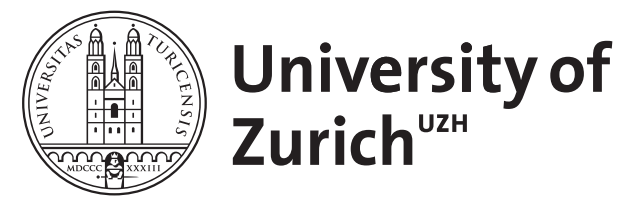

\title{
Plotin: Was fühlt der Leib? Was empfindet die Seele?
}

\author{
Caluori, Damian
}

\begin{abstract}
Thema dieses Aufsatzes ist Plotins Theorie der Emotionen, eines Themas, das in der antiken Philosophie in der Regel im Rahmen einer Handlungstheorie diskutiert wurde. So auch bei Plotin. In meinem Aufsatz wird gezeigt, wie der plotinische Leib-Seele-Dualismus im Hintergrund von Plotins Emotionstheorie steht: Leibliche Affekte werden von seelischen Emotionen unterschieden und es wird deutlich gemacht, dass das Haben einer Emotion im eigentlichen Sinn sowohl Rationalität als auch einen Leib voraussetzt. Zwei Aspekte werden besonders hervorgehoben: 1. Plotin gehört zu den Vertretern einer kognitivistischen Emotionstheorie. 2. Im Gegensatz zu vielen anderen Kognitivisten (z.B. der Stoa) macht er aber auch in einer detaillierten Analyse die Rolle des Leibes deutlich und kann diese im Rahmen seiner Theorie, im Gegensatz zu vielen anderen kognitivistischen Emotionstheoretikern, auch im Detail erklären.
\end{abstract}

DOI: https://doi.org/10.1515/9783110212198.121

Posted at the Zurich Open Repository and Archive, University of Zurich

ZORA URL: https://doi.org/10.5167/uzh-12079

Book Section

Published Version

Originally published at:

Caluori, Damian (2008). Plotin: Was fühlt der Leib? Was empfindet die Seele? In: Landweer, Hilge; Renz, Ursula. Klassische Emotionstheorien: Von Platon bis Wittgenstein. Berlin, Germany: Walter de Gruyter, 121-140.

DOI: https://doi.org/10.1515/9783110212198.121 
Plotin (205/6-270 n. Chr.) 



\section{Plotin: Was fühlt der Leib? Was empfindet die Seele?}

\section{Damian Caluori}

Plotin (205/6-270 n. Chr.) gilt als ein auf das Jenseits ausgerichteter Denker, der glaubt, der Mensch müsse dieser Welt, der Welt unserer alltäglichen irdischen Erfahrung, entfliehen, um sein wahres Glück, seine eudaimonia, zu erlangen. Dieses Bild ist nicht falsch - aber unvollständig. Tatsächlich glaubt Plotin, dass es in dieser Welt keine Güter gibt, d. h. Dinge, die für ein glückliches Leben konstitutiv sind. Der beste Zustand, in dem sich ein Mensch befinden kann und das einzige, was hinsichtlich eines guten Lebens anzustreben ist, ist das Nachdenken über die Wirklichkeit, die für Plotin, wie für die meisten Denker der späteren Antike, nicht aus der diesseitigen, sinnlich erfahrbaren Welt besteht, sondern aus einer Welt nur im Denken erfassbarer platonischer Ideen.

In diesem Bild bleibt unberücksichtigt, wie Plotin gegen radikalere Platoniker argumentiert, dass auch das diesseitige, auf die Erfahrungswelt bezogene Leben des Menschen Sinn und Funktion hat. ${ }^{1}$ Es ist daraus auch nicht ersichtlich, dass sich Plotin in den meisten seiner Schriften mit der Situation und dem Leben des Menschen im Diesseits befasst und aus platonischer Sicht Antworten auf die grundlegenden Probleme sucht, die alle antiken Philosophenschulen beschäftigt haben und von denen viele auch heute noch zum Kernbestand der Philosophie zählen. Zu diesem Kernbestand gehören heute wie damals Fragen, die das Wesen und die Funktion von Emotionen betreffen. ${ }^{2}$

1 Dazu z. B. Smith 1999; Schniewind 2003; Caluori 2005.

$2 \mathrm{Zu}$ den klassischen Abhandlungen über Plotins Emotionstheorie zählen Kristeller 1929; Blumenthal 1971, Kap. 5; Igal 1979 und Emilsson 1998. 


\section{Die affektiven Zustände des Leibes}

Wenn das Glück des Menschen im Jenseits zu suchen ist, dann scheint es zunächst nahe liegend, dass Affekte, d. h. Gefühle, mit denen wir auf Gegebenheiten und Ereignisse in der sinnlich erfahrbaren Welt reagieren, zu meiden sind. Gerade weil Affekte auf das Diesseits bezogen sind, scheint der Weise als Ideal des Menschen, der ein gutes und glückliches Leben führt, unter der oben skizzierten Voraussetzung am besten ganz ohne Affekte zu leben. Während wir uns über einen Kirschkuchen freuen oder zornig werden, wenn wir geschlagen worden sind, spielt all dies für ein auf das Jenseits bezogenes Leben keine Rolle. Solche Affekte mögen dafür sogar hinderlich sein. So scheint sich für Plotin eine Theorie der Affektlosigkeit (apatheia) nach dem stoischen Vorbild (mit seinem sprichwörtlichen stoischen Weisen) anzubieten. Eine Affektenlehre stoischen Typs würde sich nahtlos in die plotinische Konzeption des guten Lebens einfügen. Wenn man nun aber liest, was Plotin über Affekte zu sagen hat, wird schnell klar, dass es Platon ist, der bei Plotins Affektenlehre Pate gestanden hat. Dabei stellt sich die Frage, ob eine an Platon orientierte Affektenlehre mit dem stoischen Ideal der Affektlosigkeit überhaupt vereinbar ist. Die Frage stellt sich in besonderer Schärfe, weil die stoische Affektenlehre in bewusster Ablehnung der platonisch-aristotelischen Theorie entwickelt worden ist. Plotins Affektenlehre wird, wie mir scheint, am besten vor dem Hintergrund dieses Gegensatzes entwickelt.

In der Antike hat die Affektenlehre ihren Platz in der Ethik und wird üblicherweise in Abhängigkeit von der jeweiligen Güterlehre ausgestaltet. ${ }^{3}$ Platoniker und Aristoteliker gehen im Allgemeinen davon aus, dass es körperliche Güter wie Leben, Stärke und Gesundheit sowie äußere Güter wie Besitz, Freunde und ein ehrenvolles Ansehen gibt. ${ }^{4}$ Entsprechend betrachten sie das jeweilige Gegenteil (z. B. Tod, Armut und Einsamkeit) als Übel. Auf Dinge, die gut oder schlecht sind (oder zu sein scheinen), beziehen sich nun die Affekte, weshalb die Affektenlehre von der Güterlehre abhängt. Affekte gelten als vernünftig oder natürlich, wenn sie mit der Vernunft übereinstimmen. Wenn zum Beispiel ein hungriger Tiger vor mir steht, bereit, über mich herzufallen, dann habe ich allen Grund, mich zu

3 Frede 1986.

4 Neben körperlichen und äußeren gibt es auch seelische Güter. Darin sind sich Platoniker, Aristoteliker und Stoa einig, weshalb ich sie in diesem Kontext nicht weiter diskutiere. 
fürchten. Denn der Tiger ist drauf und dran, mich eines kostbaren Gutes (nämlich meines Lebens) zu berauben. Meine Furcht ist ein natürlicher oder vernünftiger Affekt, weil es für mich in dieser Situation vernünftig ist, mich zu fürchten. Wäre anstelle des Tigers ein Hase das Objekt meiner Furcht, wäre meine Furcht unnatürlich oder unvernünftig. Denn es besteht kein Grund anzunehmen, dass der Hase mich eines Gutes berauben oder mir ein Übel antun wird. Allgemeiner gesagt ist es vernünftig, sich zu Gütern hingezogen zu fühlen, sich an ihrem Besitz zu erfreuen und ihren Verlust zu fürchten bzw. zu bedauern und bei Übeln das jeweilige Gegenteil zu empfinden.

Diese Affekte haben nun in der platonisch-aristotelischen Tradition ihren Platz in einem oder zwei irrationalen Seelenteilen, die zusammen mit der Vernunft die Seele konstituieren, und die - im Idealfall - so unter der Kontrolle der Vernunft stehen, dass alle Affekte, die auftreten, natürlich oder vernünftig sind. ${ }^{5}$ Bei Platon sind die irrationalen Seelenteile das Mutartige (thymoeides) und das Triebhafte (epithymêtikon). Gemäß der platonischaristotelischen Konzeption ist es Teil eines gelungenen Lebens, die den jeweiligen Lebenssituationen angemessenen vernünftigen Affekte zu haben und nie auf der Vernunft entgegengesetzte Weise affiziert zu werden, d. h. nie Affekte zu haben, für deren Auftreten es keinen vernünftigen Grund gibt. In der Antike wurde diese Verfassung als ,maßvolle Affizierbarkeit' (metriopatheia) bezeichnet. ${ }^{6}$

Die Stoa hat die platonisch-aristotelische Güterlehre radikal abgelehnt, weshalb auch ihre Affektenlehre wesentlich verschieden ist. Die Stoiker behaupten nämlich, es gebe nur ein Gut, die Tugend und nur ein Übel, die Untugend - beides Eigenschaften der Seele. Es gibt also im Stoizismus weder äußere noch körperliche Güter oder Übel. Alles außer der Tugend und der Untugend ist weder gut noch schlecht, sondern indifferent (adiaphoron). Affekte, die vermeintliche äußere oder körperliche Güter oder Übel zum Objekt haben, sind zu meiden, und entsprechend ist der stoische Weise frei von ihnen (apathês). Dadurch verliert auch der platonischaristotelische irrationale Seelenteil seine Funktion. Die stoische Seele hat keinen irrationalen Teil - alle Affekte sind Affekte der Vernunft. Sie sind

$5 \quad$ Ergänzend ist zu bemerken, dass bei Platon und bei Aristoteles auch der rationale Seelenteil Emotionen hat und von sich aus fähig ist, Handlungen zu motivieren. Siehe dazu Cooper 1984.

6 Z. B. Alexander v. Aphrodisias, In Topica, 239, 6; Wallies und Porphyr, Sententiae, 32; dazu Dillon 1983. Für eine Abhandlung zum Thema Emotionen in der aristotelischen Tradition siehe Aspasios, In Ethica Nicomachea, 42, 27-47, 2. 
nämlich gemäß der intellektualistischen (oder kognitivistischen) stoischen Position nichts anderes als falsche Urteile.

Wo steht nun Plotin in dieser Debatte? Wie bereits eingangs erwähnt, scheint der stoische Weise mit seiner Affektlosigkeit dem plotinischen Ideal besser zu entsprechen als der platonisch-aristotelische, der natürliche oder vernünftige Affekte empfindet. Von diesem Standpunkt aus mag es überraschen, dass Plotin von einem affektiven (pathêtikon) Teil der Seele spricht und ihm eine entscheidende Rolle in seiner Affektenlehre zuspricht. ${ }^{7} \mathrm{Zu}$ diesem affektiven Teil gehören nun gemäß Plotin das Muthafte (thymoeides) und das Triebhafte (epithymêtikon), also zwei Vermögen, deren Entsprechungen bei Platon die irrationalen Seelenteile sind. ${ }^{8}$

$\mathrm{Da}$ nun die Seele einen affektiven Teil hat, würde man erwarten, dass selbst die Seele eines Weisen, wie in der platonisch-aristotelischen Tradition, Affekten unterliegt. Plotin diskutiert die Frage, ob sich dies so verhält, in einem Traktat mit dem Titel Über die Unaffizierbarkeit der unkörperlichen Dinge (Enn. III 6). Wie der Titel suggeriert, ist Plotin der Ansicht, dass nur Körper etwas erleiden können. ,Erleiden' ist hier in einem sehr weiten Sinn gemeint (und nicht auf Affekte beschränkt), und der Traktat ist ein Beitrag Plotins zur antiken Diskussion von Tun (poiein) und Erleiden (paschein), zweier Begriffe, mit deren Hilfe Probleme erörtert wurden, die wir heute unter dem Stichwort ,Kausalität' behandeln: ${ }^{9}$ Jede Veränderung hat zwei Aspekte, einen aktiven und einen passiven, und entsprechend gibt es etwas, das als Wirkursache fungiert, und etwas, an dem die Veränderung stattfindet (oder das die Veränderung erleidet). ${ }^{10}$ Plotins Behauptung ist nun die, dass nur Körper etwas erleiden, d. h. durch eine Wirkursache verändert werden können. Da die Seele bei Plotin, wie allgemein im Platonismus, unkörperlich ist, kann sie auch nichts erleiden.

Zwar wird dasselbe griechische Wort, pathos, sowohl für den Begriff des Erleidens in kausalitätstheoretischen Diskussionen als auch für Affekte wie Freude oder Schmerz verwendet, daraus dürfen wir aber natürlich

7 Enn. III 6, 4, $1 \mathrm{ff}$. Von einem affektiven Teil der Seele ist bereits bei Aristoteles, Politika 1254b8, die Rede.

8 Platon führt die zwei erwähnten irrationalen Seelenteile in Staat IV ein. Vor Plotin fasst bereits Alkinoos, Didaskalikos, Kap. 24 die irrationalen platonischen Seelenteile in einem affektiven Seelenteil (pathêtikon) zusammen.

9 Die erste systematische Abhandlung dieses Themas ist wohl Aristoteles, De Generatione et Corruptione I 7.

10 Siehe z. B. Sextus Empiricus, Grundriss der pyrrhonischen Skepsis III 14 und Clemens von Alexandrien, Stromata I 17, 82, 3. 
nicht unmittelbar schließen, dass Affekte zum Erleiden zu zählen sind. Der Ausdruck ,pathos könnte auch homonym einerseits für Affekte und andererseits, in ganz anderer Bedeutung, für das Erleiden verwendet werden. Trotzdem scheint die Annahme, dass Affekte im Sinne von Emotionen einen passiven Aspekt aufweisen, der dem des Erleidens entspricht, gut zu unseren Intuitionen zu passen. Auch die Vertreter eines irrationalen Seelenteils scheinen dieser Meinung gewesen zu sein, was sich möglicherweise darin zeigt, dass sie annahmen, Affekte ließen sich nicht eliminieren, sondern bestenfalls von der Vernunft kontrollieren. Aber wie dem auch sei: Plotin jedenfalls versteht Affekte als eine Art von Widerfahrnis, wie seine Diskussion in Enn. III 6, 4 zeigt, und er besteht darauf, dass der Seele, da sie unkörperlich ist, nichts widerfährt. A fortiori gilt, dass sie keinen Affekten unterworfen ist. Selbst der affektive Teil der Seele wird von nichts affiziert und ist daher auch nicht das Subjekt solcher Affekte wie Freude, Trauer, Lust oder Schmerz (ebd.). Anstatt Affekte zu erleiden, hat dieser Teil der Seele bei der Entstehung von Affekten eine aktive Rolle zu spielen. ${ }^{11}$ Er ist - in einem gewissen Sinn - die Ursache der Affekte.

In welchem Sinn? Plotin vergleicht den affektiven Teil der Seele mit dem Teil, der für das Wachstum des Körpers zuständig ist (ebd.). Dieser Teil ist dafür verantwortlich, dass der Körper wächst, wenn gewisse Bedingungen erfüllt sind. Zu diesen Bedingungen zählen zum Beispiel, dass der Körper noch nicht ausgewachsen ist und dass ihm ausreichend Nahrung zugeführt wird. Entsprechend wird der affektive Teil der Seele dafür zuständig sein, dass, wenn bestimmte Bedingungen erfüllt sind, das Lebewesen entsprechende Affekte empfindet.

Plotin diskutiert dieses Beispiel, um durch Analogie zu zeigen, dass ein Seelenteil durch das Bewirken einer bestimmten Veränderung nicht notwendigerweise selbst einer Veränderung unterliegt. Wie der Seelenteil, der Wachstum bewirkt, nicht selbst wächst, so wird der Seelenteil, der Affekte bewirkt, nicht selbst affiziert. Es ist aber auch klar, dass es etwas geben muss, dass der Veränderung, welche die Seele bewirkt, unterworfen ist. Beim Beispiel des Wachstums ist dies natürlich der Körper des Lebewesens. Veränderungen, die der affektive Seelenteil bewirkt, finden nun gemäß Plotin ebenso wie das Wachstum im Leib statt.

Dass der Seelenteil, der für das Wachstum zuständig ist, und der affektive Seelenteil beide im Körper des Lebewesens aktiv sind, ist kein Zu-

11 Zur Rolle des affektiven Seelenteils bei der Entstehung von Affekten siehe Emilsson 1998. 
fall. Plotin ist nämlich der Auffassung, dass es sich nicht um zwei verschiedene Seelenteile handelt, sondern nur um verschiedene Funktionen desselben Seelenteils. Diesen bezeichnet er oft mit den auf Aristoteles zurückgehenden Ausdrücken als zeugenden (gennêtikon) oder als vegetativen (phytikon) Seelenteil. ${ }^{12}$ Aristoteles verwendet diese Ausdrücke zur Bezeichnung der Seelenfunktionen, die für das Leben eines körperlichen Lebewesens grundlegend sind: Ernährung, Wachstum, Fortpflanzung - also Funktionen, die für den Erhalt und die Reproduktion des Körpers besorgt sind.

Nun ist es klar, dass Affekte nicht in jedem Körper auftreten. Stühle ärgert es nicht, wenn man sich auf sie setzt, und sie sind nicht erleichtert, wenn man wieder aufsteht. Sie empfinden gar nichts. Nur lebende Körper haben Affekte. Um den Unterschied zwischen Körpern von Lebewesen und solchen Körpern wie Steinen oder Stühlen klar zu markieren, verwendet Plotin für erstere den technischen Ausdruck „so-beschaffener Körper" (toionde sôma). Wie bereits Theiler bemerkt hat, geht auch dieser Ausdruck auf Aristoteles zurück, ob dieser ihn nun auch bereits technisch verwendet hat oder nicht. ${ }^{13}$ Aristoteles bezeichnet in De anima einen Körper als so-beschaffen, wenn er über die grundlegenden Lebensfunktionen wie Selbsternährung und Wachstum verfügt. Auch bei Plotin ist ein sobeschaffener Körper einer, der über die grundlegenden Lebensfunktionen verfügt und in dem somit der vegetative Seelenteil aktiv ist. Wie oben gesagt, ist der affektive Seelenteil identisch mit dem vegetativen. Wir nennen den vegetativen Seelenteil affektiv, wenn wir von seinen affektiven Funktionen reden. Also ist in einem so-beschaffenen Körper, einem Leib, auch ein affektives Vermögen vorhanden.

Für unseren Zusammenhang ist wichtig, dass Affekte für Plotin Aktivitäten sind, die in den grundlegenden Funktionen des Leibes angelegt sind. Sie sind also für Plotin in doppeltem Sinne Affekte des Leibes: Sie treten im Leib auf und dienen dessen Erhaltung und Reproduktion. Hunger, Angst und Schmerz sind klare Beispiele dafür, wie wichtig Affekte für das Leben eines körperlichen Lebewesens sind. Wir sehen also bereits hier, wie Plotin die eingangs aufgestellte Aporie auflöst. Affekte sind zwar

12 Die Ausdrücke kommen z. B. vor bei Aristoteles: Nikomachische Ethik 1102a32; De generatione animalium 735a17; bei Plotin: Enn. I 1, 8, 21; IV 4, 27,1f.; IV 4, 28, 16. Das Vermögen wird von Aristoteles in De anima II 4 diskutiert.

13 Aristoteles, De Anima 403a26; 412a11f.; 16f.; b11; 16; 27; 414a22. Theiler: Anmerkung zu Enn. II 3, 9, 12 in Plotin 1956-1971. 
nicht Teil eines guten Lebens, sie sind aber trotzdem notwendiger und nützlicher Bestandteil eines jeden leiblichen Lebens.

In einem Exkurs in Enn. IV 4 (Kap. 28 und 29) diskutiert Plotin eine ganze Reihe von Affekten und klassiert sie unter das Mutartige (thymoeides) und das Triebhafte (epithymêtikon). Affekte treten in verschiedenen Teilen des Körpers auf. Plotin lokalisiert Affekte, die zum Mutartigen gehören, tendenziell im Bereich des Herzens und solche, die zum Triebhaften gehören, tendenziell im Bereich der Leber. Die Lokalisierung ist so zu verstehen, dass ein Affekt der jeweiligen Art in der Regel im jeweiligen Organ seinen Ursprung hat. So findet in der Leber der größte Teil der triebhaften und im Herzen der größte Teil der mutartigen Aktivität statt. ${ }^{14}$ Es gibt aber auch Affekte, die in anderen Körperteilen ihren Platz haben. So lokalisiert Plotin die sexuelle Lust in den Geschlechtsorganen (Enn. IV 4, 28). Schmerzen treten an den Stellen im Körper auf, die verletzt worden (oder erkrankt) sind. Der affektive Teil der Seele ist also nicht bloß auf die zwei Zentralorgane Herz und Leber beschränkt, sondern im ganzen Leib aktiv.

Die Tatsache, dass alle Affekte solche des Leibes sind, impliziert nicht, dass sie rein physikalische Ereignisse sind. Die Idee, dass sich Körper von Lebewesen nicht prinzipiell von anderen Körpern unterscheiden, ist wohl erst cartesisch, die Folge eines bestimmten Verständnisses der Körperwelt, gemäß welchem alle körperlichen Veränderungen in gleicher Weise durch die Physik erklärt werden können. ${ }^{15}$ Dass zumindest Plotin weit davon entfernt ist, ein solch cartesisches Verständnis zu teilen, wird schon daraus klar, dass er, worauf ich bereits oben hingewiesen habe, auf den (wie er es sieht) fundamentalen Unterschied zwischen leblosen Körpern (wie Stühlen und Steinen) und belebten Körpern großen Wert legt.

Dass Affekte aber durchaus auch physikalische Vorgänge und eng mit körperlichen Zuständen und Dispositionen verwoben sind, wird von Plotin nicht bezweifelt. Das zeigt seine Diskussion des Zorns in Enn. IV 4, 28: Das Blut und die Galle um das Herz kochen, wenn man zornig ist. Derselbe Mensch wird schneller zornig, wenn er krank oder hungrig, als wenn er satt oder gesund ist. Dass es aber auch Unterschiede zwischen

14 Die Lokalisierung der zwei Affektarten in Herz bzw. Leber entspricht Platon, Timaios 69c-72d.

15 Siehe vor allem Descartes' Traité de l'Homme und La Description du corps humain et de toutes ses fonctions. Dazu und allgemein zur frühneuzeitlichen Idee des menschlichen Körpers als Maschine vgl. Giglioni 1995 und Des Chene 2001. 
den Menschen gibt, zeigt sich daran, dass Menschen mit wenig Galle milde sind und kaum in Rage geraten. ${ }^{16}$

Dem lebenden Körper ist aber nicht nur das Kochen des Blutes und der Galle als physiologischer Prozess zuzuschreiben, sondern auch das Gefühl des Zorns. Mit „Gefühl“ meine ich den qualitativen oder phänomenalen Aspekt des Affekts. ${ }^{17}$ Dass der Leib auch fühlt, ist eben gerade darin begründet, dass er nicht ein Körper wie ein Stuhl ist, sondern ein lebender Körper. Entsprechend sagt Plotin in Enn. IV 4, 19 über den Schmerz, dass, wenn der Körper bei einer Operation aufgeschnitten wird, die Gewebemasse (ogkos) geschnitten wird - aber eben nicht nur: Es findet auch eine Schmerzempfindung im Gewebe statt, denn das Gewebe ist lebendes Gewebe. Im selben Kapitel betont Plotin, dass, wenn man sagt, der Mensch habe am Finger Schmerzen, dies eine uneigentliche Art zu reden sei, wie wenn man (metonymisch) einen Menschen strahlend nennt, wobei eigentlich sein Gesicht strahlt. In Wahrheit, so Plotin, schmerzt der Finger.

Es ist bemerkenswert, dass Plotin in seiner Rede von Affekten oft von deren Lokalisierung spricht. Der Grund dafür ist darin zu finden, dass Affekte - buchstäblich gesprochen - im Körper stattfinden. Sie sind aber keine rein physiologischen Ereignisse (in unserem modernen Sinn), sondern haben wesentlich auch einen qualitativen oder phänomenalen Aspekt. Das Gefühl ist vom physiologischen Vorgang nicht zu trennen: Beide zusammen konstituieren den Affekt.

\section{Was empfindet die Seele?}

Vorerst eine terminologische Bemerkung. Um den Begriff der leiblichen von dem der seelischen pathê bei Plotin zu unterscheiden, nenne ich erstere „Affekte“ und letztere „Emotionen“. Das scheint mir gerechtfertigt, weil Plotin das Wort pathos, wie wir gleich sehen werden, entsprechend in zwei verschiedenen Bedeutungen verwendet.

16 Die Tatsachen, dass (a) dieselbe Person zu verschiedenen Zeiten vom selben Affekt verschieden affiziert werden kann, ohne dabei ihre Überzeugungen geändert $\mathrm{zu}$ haben, und (b) verschiedene Personen mit denselben Überzeugungen von demselben Affekt verschieden affiziert werden können, kann Plotin im Gegensatz zum klassischen (chrysippeischen) Stoizismus erklären.

17 Ich möchte terminologisch zwischen „Gefühl“, „Affekt“ und, wie wir sehen werden, „Emotion“ unterscheiden. 
Unsere bisherige Diskussion war ganz auf den Leib beschränkt. Die Seele, soweit wir sie bis jetzt betrachtet haben, hatte bloß die Funktion, durch den affektiven Teil Affekte im Leib zu bewirken. Was heißt das nun für die Seele? Ist sie ganz ohne Emotionen? Verfügt sie über eine andere Art von Handlungsmotivation oder ist ihre ganze Tätigkeit ausschließlich kognitiv? Auch zu den Affekten stellen sich weitere Fragen: Reichen die Affekte des Leibes aus, um den ganzen Reichtum menschlicher Emotionen zu erklären? Die letzte dieser Fragen ist vor allem vor dem stoischen Hintergrund von großer Bedeutung. Denn die Stoiker haben nachdrücklich betont, dass Emotionen kognitiven Gehalt haben: Sie sind ihnen gemäß Urteile der Vernunft. Es ist gerade dieser Aspekt, der die stoische Position für manche Philosophen heute wieder attraktiv macht. ${ }^{18}$

Nehmen wir zunächst an, Plotin habe die stoische Position rundweg abgelehnt und seine ganze Emotionstheorie auf eine Theorie der leiblichen Affekte beschränkt. Wäre dies der Fall, wäre jede Seele zu jedem Zeitpunkt frei von jeglichen Emotionen. Um zu klären, ob dies tatsächlich Plotins Position ist, wollen wir zunächst weiter untersuchen, welche Rolle die Seele bei Affekten spielt.

Wie eingangs erwähnt, spielen Affekte traditionell für Handlungen eine entscheidende Rolle, weil ihre Objekte (wirkliche oder vermeintliche) Güter und Übel sind. Die meisten Menschen streben nach Dingen wie Ansehen, Gesundheit und Freunden, weil sie diese Dinge für Güter halten (,zu Recht“, sagen traditionelle Platoniker und Aristoteliker - „zu Unrecht", antworten Stoa und Plotin). Ist es nun ausschließlich der Leib, der uns dazu motiviert, nach solchen Dingen zu streben und ihr jeweiliges Gegenteil zu meiden? Falls ja: Welche Rolle spielt dann die Seele bei der Handlungsmotivation?

Bei vielen der (scheinbaren) Güter, nach denen wir streben, erfordert schon allein der Versuch, sie zu erlangen, komplexe kognitive Aktivität, wenn er nicht ganz hoffnungslos sein will. Man muss planen, kalkulieren, diverse Faktoren in Betracht ziehen usw. Es ist klar, dass an solchen Handlungen die Seele als kognitives Vermögen beteiligt sein muss, schon allein deshalb, weil auch viele Überzeugungen in diejenigen Prozesse involviert sind, die zu einer Handlung führen. Außerdem wird es der Leib einer Person kaum alleine schaffen, zornig zu werden, wenn zum Beispiel

18 So bezeichnet z. B. Nussbaum 2001, 4 aus diesem Grund ihre eigene Position als „neo-Stoic“. Das stoische Ideal der Affektlosigkeit hingegen findet heute kaum noch Freunde. 
ein Witz auf ihre Kosten gemacht wird. Ihr Leib wird den Witz nicht verstehen und noch weniger, dass er auf sie gemünzt ist. Ohne kognitive Aktivität kocht in diesem Fall weder Blut noch Galle.

Außerdem muss die Seele in der Lage sein, zur Kenntnis zu nehmen, in welchen affektiven Zuständen sich der Leib befindet. Denn es gehört zu den Aufgaben der Seele, sich um den Leib zu kümmern. Wenn der Leib zum Beispiel Nahrung braucht und somit im affektiven Zustand des Hungers ist, dann muss die Seele fähig sein, dies irgendwie zu bemerken. Damit sie sich um den Körper kümmern kann, muss sie bedenken können, was ihm in konkreten Situationen nützt und schadet - und dazu muss sie nicht nur die aktuelle Umgebung wahrnehmen (sind Beeren oder Tiger in der Nähe?), sondern auch den Zustand des Körpers (Braucht er Nahrung? Hat er sich verletzt?).

In seiner Diskussion vom schmerzenden Finger sagt Plotin entsprechend, dass der Affekt zwar im Leib stattfinde, die Seele ihn aber zur Kenntnis nehme (Enn. IV 4, 19). Auch beim Auftreten einer Begierde im Leib wird die Seele mittels einer Vorstellung (phantasia) darüber informiert, wobei Plotin die Vorstellung der Begierde mit einer Wahrnehmung vergleicht (Enn. IV 4, 17). ${ }^{19}$ An anderen Stellen erklärt Plotin ausdrücklich, dass die Seele die Affekte des Leibes wahrnebme. ${ }^{20}$ Die Seele steht also auf diese Weise mit dem Leib in einer ähnlichen Beziehung wie mit der AuBenwelt.

Wie wir bereits im ersten Teil gesehen haben, kann die Seele mittels ihres affektiven Teils auf die affektiven Zustände des Körpers einwirken. Dies wird in Plotins Diskussion des Zorns in Enn. IV 4, 28 deutlich:21 Zorn kann seinen Ursprung im Leib haben, nämlich dann, wenn der Leib etwas erleidet, so dass, als Folge davon, das Blut und die Galle um das Herz erhitzt werden und das Gefühl des Zorns aufkommt. In diesem Fall, den wir oben schon betrachtet haben, nimmt die Seele den Zorn des Leibes wahr. Es gibt aber auch viele Fälle, bei denen wir zornig werden, obwohl dem Leib nichts geschehen ist. Wir zürnen z. B. über Dinge, die unseren Freunden zustoßen und überhaupt über ungebührliche Handlungen (ebd.). In solchen Fällen ist nicht der Leib der Ursprung des Zorns, sondern die Seele. Dabei nimmt die Seele wahr (oder zumindest scheint es der

19 Ich werde auf den Begriff der Vorstellung zurückkommen.

20 Enn. I 1, 1, 6; Enn. III 6, 1, 2; Enn. IV 3, 26, 8.

21 Für eine analoge Erklärung des Auftretens von Furcht siehe Enn. III 6, 4. Dazu Fleet in Plotinus 1995, 115-132 und Rehm 1997. 
Seele), dass ein Unrecht geschehen ist - auch ein Unrecht, welches nicht ihren Leib betrifft. Sie bringt den Leib in einen zornigen Zustand und macht ihn, wie Plotin sich ausdrückt, zu ihrem „Kampfgefährten“ (ebd.).

Die Seele nimmt also die affektiven Zustände des Körpers wahr, kann diese aber auch bewirken. Auch diese Relation zwischen Seele und Leib findet eine Entsprechung im Verhältnis zwischen Seele und Außenwelt. ${ }^{22}$

Dass die Seele auf diese Weisen mit dem Leib interagiert, impliziert jedoch nicht, dass sie selbst Emotionen empfindet. Vielleicht ist das Engagement der Seele ausschließlich kognitiv, und sie selbst empfindet dabei keine Emotionen. Und es gibt eine Stelle bei Plotin, die man leicht auf diese Weise interpretieren kann. In Enn. IV 4, 18 wird nämlich gesagt, Schmerz und Lust des Körpers resultiere für die Seele in einer affektlosen Kenntnisnahme (eis gnôsin apathê).

Nun kann aber die Ansicht, die Seele sei bloß kognitiv aktiv, nicht diejenige Plotins sein. Das wird aus seiner Diskussion der Konflikte zwischen Leib und Seele deutlich. ${ }^{23}$ Die Seele kann von den Begierden und Ängsten des Leibes überwältigt werden und sie sich zu eigen machen. Sie kann sich ihnen aber auch entgegenstellen, sodass man, obwohl einem angesichts eines leckeren Kuchens das Wasser im Mund zusammenläuft, seine Diät fortsetzt oder trotz zitternder Glieder und schwitzender Hände nicht davonrennt. Die Konflikte zwischen Leib und Seele können nicht erklärt werden, wenn die Seele nur kognitiv aktiv ist und selbst keine motivationale Kraft besitzt. Natürlich reicht das Zugeständnis, dass die Seele ihre eigene motivationale Kraft hat, noch nicht dafür aus, zu schließen, dass sie emotional berührt wird. Vielleicht nimmt sie die Freuden und Leiden des Leibes ja unbewegt zur Kenntnis und setzt sich dem Leib durch eine rein rationale Motivation entgegen. Wenn dies der Fall wäre, würde die Seele selbst dann unbewegt bleiben, wenn ihr Leib auf die Folterbank gespannt würde. Nach dieser Interpretation würden sich die Qualen des Leibes für die Seele bloß in einem desinteressierten, leidenschaftslosen Zurkenntnisnehmen erschöpfen.

Vielleicht ist es problemlos, dies für die Seele eines Weisen anzunehmen. Tatsächlich scheint Plotin der Meinung zu sein, dass es ein Zeichen

22 Plotins Diskussion der Interaktion zwischen Leib und Seele zeigt, wie weitgehend der Leib für die Seele der Außenwelt angeglichen ist, ohne jedoch - wie wir im ersten Teil gesehen haben - schon cartesisch etwas ganz Äußerliches zu sein. Der plotinische Leib-Seele-Dualismus ist vom cartesischen verschieden, trägt aber ähnliche Züge.

Enn. II 3, 9; Enn. III 1, 3, 24; Enn. IV 8, 1, $24 f f$. 
der Weisheit ist, klar zwischen den Angelegenheiten der Seele und denen des Leibes zu unterscheiden und sich letztere nicht zu eigen zu machen. In Enn. I 4, 4 sagt er, der Weise kümmere sich zwar um den Leib, der mit ihm verknüpft sei, dieser habe aber sein eigenes Leben, und das sei nicht das Leben des Weisen. Denn das Leben des Weisen ist das Leben seiner Seele. Der Weise mag also von den Kalamitäten, die seinen Leib befallen, emotional ganz unberührt bleiben.

Aus denselben Stellen wird aber auch klar, dass dies für den Nichtweisen - und das heißt für uns alle - nicht gilt. Unsere Seele, im Gegensatz zu der des Weisen, wird bewegt oder gar erschüttert, wenn der Leib schmerzt, wenn Freunde sterben, wenn unsere Stadt niedergebrannt wird, wenn unsere Töchter von den Feinden verschleppt werden. ${ }^{24}$ Diese seelischen Erschütterungen und Leiden sind nach Plotin eben gerade darin begründet, dass wir glauben, es sei ein Übel, wenn einem solcherlei zustößt. Für unseren momentanen Zusammenhang ist aber nur wichtig zu sehen, dass Plotin durchaus der Meinung ist, dass wenigstens Seelen von Nichtweisen - und damit zumindest wir alle - Emotionen empfinden.

Das wird auch aus Enn. I 1 klar. Die Schrift beginnt mit folgenden Worten: „Lust und Unlust, Furcht und Mut, Begierde und Abneigung, und der Schmerz, wer ist ihr Träger?" Es stellt sich also die Frage nach dem Träger, dem Subjekt, von Emotionen. In der Folge ist die Diskussion in diesem Traktat aber nicht auf Emotionen beschränkt, sondern beschäftigt sich auch mit der Frage nach dem Träger von Wahrnehmungen, Meinungen und diskursivem Denken und geht davon aus, dass all diese Aktivitäten solche desselben Subjekts sind. Allein schon dieser Kontext macht es deutlich, dass Plotin nicht den Leib mit seinen Affekten im Auge hat, sondern die Seele. Denn Wahrnehmung, Meinungen und diskursives Denken gehören zweifellos zur Seele und nicht zum Leib. Da Emotionen zum selben Subjekt wie diese gehören, muss also auch die Seele Emotionen besitzen. Da Affekte zum Leib gehören, können Emotionen aber keine Affekte sein. Was sind sie dann?

Um diese Frage zu beantworten, möchte ich auf die früher aufgestellte Behauptung zurückkommen, gemäß der die Seele die Affekte des Leibes wabrnimmt. Ich werde nun dafür argumentieren, dass es eine Klasse von Emotionen gibt, die eine bestimmte Art von Wahrnehmung sind. Bei solchen Emotionen geht die Gefühlsfärbung und die motivationale Kraft auf

24 Siehe Enn. I 4, 7. 
einen entsprechenden leiblichen Affekt zurück. ${ }^{25}$ Ich werde nicht behaupten, dass alle Emotionen zu dieser Klasse gehören und am Ende kurz auch auf Emotionen eingehen, die nichts mit dem Leib zu tun haben.

In seinem grundlegenden Buch zu Plotins Wahrnehmungstheorie zeigt Emilsson, dass Plotin zwischen dem sinnlichen Affekt und der eigentlichen Wahrnehmung unterscheidet. ${ }^{26}$ Der sinnliche Affekt findet im Leib statt, während die eigentliche Wahrnehmung ein Wahrnehmungsurteil der Seele ist. Emilssons Interpretation gemäß bestimmt Plotin den sinnlichen Affekt als Anschauung (engl. sensation). Emilsson erklärt dies mit folgendem Beispiel: ${ }^{27}$ Nehmen wir an, ich sehe ein grünes Feld, hebe meine Augen und sehe den blauen Himmel. Der Wechsel vom Sehen des Feldes zum Sehen des Himmels involviert einen Wechsel von einem Wahrnehmungsurteil („Ich sehe das grüne Feld.“) zu einem anderen („Ich sehe den blauen Himmel."). Emilsson argumentiert nun dafür, dass diesem Wechsel ein anderer Wechsel zugrunde liegt, bei dem ich ein rein passiver Rezipient bin. Wenn man jegliche Begrifflichkeit von meiner visuellen Erfahrung abzieht, bleibt immer noch etwas übrig. Emilsson vergleicht das, was übrig bleibt, mit der Erfahrung eines noch nicht der Sprache mächtigen Kleinkindes, das seine Augen vom grünen Feld zum blauen Himmel hebt. Was das Kleinkind wahrnimmt, kann man wohl als reine Farberfahrung bezeichnen - eine Erfahrung, die frei von jeglicher Begrifflichkeit ist. Auf diese Weise würde jedenfalls Plotin den eben beschriebenen Wechsel nach Emilssons Deutung erklären. Diese rein passive Erfahrung nennt Emilsson nun Anschauung (sensation) und identifiziert sie mit dem Affekt des Leibes. Auch hier ist also der Affekt nicht bloß ein physiologischer Vorgang, sondern auch ein phänomenaler: Das Kleinkind sieht (vorbegrifflich) Grün und Blau. Die eigentliche Wahrnehmung aber ist ein Urteil der Seele. Die Seele urteilt, dass das Feld grün ist und dass der Himmel blau ist.

25 Das impliziert nicht, dass sich solche Emotionen ausschließlich auf leibliche Zustände beriehen, wie das obige Beispiel des Zorns zeigt. Ich kann nämlich zornig darüber sein, dass einem Freund ein Unrecht geschehen ist. Auch in solchen Fällen kommt jedoch die Gefühlsfärbung vom Leib.

26 Emilsson 1988. Für unseren Kontext sind vor allem Kap. IV und VII wichtig. Dass Plotin Anschauung (engl. sensation) und Wahrnehmung (engl. perception) unterscheidet, hat bereits Dodds (in seinem Kommentar zu Schwyzer 1960, 385) bemerkt, aber erst Emilsson hat den Unterschied systematisch ausgearbeitet.

27 Emilsson 1988, 83f. 
Wie bei der Stoa besteht also eine Wahrnehmung der Seele in einem Urteil. Gemäß der stoischen Erkenntnistheorie heißt das: in einer $\mathrm{Zu}-$ stimmung zu einer Vorstellung. Das ist nur möglich, wenn menschliche Vorstellungen propositionalen Gehalt haben. Eine Vorstellung zu haben heißt, dass sich einem ein Sachverhalt auf gewisse Weise darstellt. Wenn man sieht, dass die Magnolie vor dem Haus blüht, hat man den Eindruck oder die Vorstellung, dass die Magnolie vor dem Haus blüht (wobei „Die Magnolie blüht vor dem Haus.“ der propositionale Gehalt der Vorstellung ist). Die Tatsache, dass zwei Vorstellungen genau denselben propositionalen Gehalt haben, impliziert aber nicht, dass es genau dieselben Vorstellungen sind. Derselbe propositionale Gehalt kann mir nämlich ganz verschieden erscheinen. Die wahre Vorstellung, dass die Magnolie vor dem Haus blüht, ist eine andere, je nachdem, ob ich es mit eigenen Augen sehe, ob es mir jemand erzählt oder ob ich es geträumt habe. Außerdem wird das Herz eines Blumenfreundes höher schlagen, wenn er hört, dass die Magnolie vor dem Haus blüht, während derselbe Sachverhalt einer weit fortgeschrittenen Mystikerin ganz indifferent erscheinen mag. Entsprechend werden die Vorstellungen von Blumenfreund und Mystikerin trotz desselben propositionalen Gehaltes sehr verschieden sein.

Während uns Vorstellungen unwillkürlich gegeben sind, liegt die $Z u$ stimmung zu ihnen in unserem Ermessen. Nur wenn wir eine Vorstellung akzeptieren, glauben wir, dass sich die Sache so verhält, wie wir sie uns vorstellen (oder: wie sie sich uns darstellt). Wir können aber auch Vorstellungen haben, denen wir nicht zustimmen. Der halb ins Wasser getauchte Stab erscheint einem immer noch als gebrochen, nachdem man sich überzeugt hat, dass er nicht gebrochen ist. Da einem in diesem Beispiel der Stab als gebrochen erscheint, hat man die Vorstellung, dass er gebrochen ist. Man wird dieser Vorstellung aber nicht zustimmen, wenn man sich davon überzeugt hat, dass sie falsch ist. ${ }^{28}$

Wie die Stoa hält auch Plotin menschliche Seelen für rational und entsprechend haben menschliche Vorstellungen propositionalen Gehalt. Eine Wahrnehmung ist die Zustimmung zu einer entsprechenden Vorstellung. Wenn wir nun Plotins Behauptung, Emotionen seien eine Art Wahrnehmung, ernst nehmen - und ich sehe keinen Grund, warum wir dies nicht

28 Sowohl in der stoischen Ethik und Erkenntnistheorie als auch in der plotinischen Ethik ist die Unterscheidung zwischen unwillkürlich gegebener Vorstellung und der Zustimmung zu ihr, welche allein in unserem Ermessen liegt, von entscheidender Bedeutung. Für die Stoa siehe Görler 1977, Arthur 1983 und Frede 1983. 
tun sollten -, dann trifft das, was eben zu Plotins Wahrnehmungstheorie gesagt wurde, auch auf solche Emotionen zu.

Aufgrund der obigen Besprechung leiblicher Affekte ist klar, dass Affekte nicht bloß physiologische Veränderungen sind, sondern wesentlich auch Gefühle. Diese Gefühle entsprechen nun der Anschauung in der Sinneswahrnehmung. Die Seele hat nun unwillkürlich eine Vorstellung. Diese Vorstellung hat propositionalen Gehalt, nimmt aber in der Regel auch den Gefühlsaspekt des leiblichen Affekts auf - genauso wie die Vorstellung einer sinnlichen Wahrnehmung die farblichen (oder sonstigen qualitativen) Aspekte der Anschauung aufnimmt. Wenn der Finger schmerzt und die Seele dies wahrnimmt, wird sie also die Vorstellung haben, dass der Finger schmerzt. Diese Vorstellung hat propositionalen Gehalt - aber nicht nur: Auch das Schmerzgefühl ist Teil der Vorstellung.

In Enn. VI 8, 3 erklärt Plotin genau dies: Je nachdem, in welchem Zustand sich ihr Körper befindet, hat die Seele verschiedene Vorstellungen. ${ }^{29}$ Dass es dabei nicht bloß um Propositionen geht, die den Zustand des Leibes wiedergeben, wird wieder aus Plotins Diskussion des Zorns klar. Wir haben bereits gesehen, dass Zorn sowohl von der Seele als auch vom Leib ausgehen kann. Wenn der Zorn im Leib seinen Anfang nimmt, dann „tritt eine Wahrnehmung ein und die Vorstellung gibt der Seele Anteil an der Disposition des so bestimmten Leibes [...]“ (Übersetzung Harder). Wir erfahren im selben Abschnitt außerdem, dass der vom Leib ausgehende Zorn die Seele mittels einer Vorstellung auf seine Seite reißt. Die Tatsache, dass der leibliche Zorn die Seele mittels einer Vorstellung zu etwas hinreißen kann, zeigt, dass die Vorstellung nicht bloß eine Proposition ist, sondern auch einen motivationalen Aspekt aufweist: In der Vorstellung bleibt das Gefühl des Zorns erhalten, es wird aber begrifflich gefasst.

Die Qualität der Vorstellung hängt also vom Affekt ab, von dem man eine Vorstellung hat. Dies ist nicht überraschend. Es ist aber wichtig zu sehen, dass sie nicht nur vom Affekt abhängt, sondern auch davon, wie sich die Seele zum Affekt verhält. Dies wiederum hängt von den Überzeugungen ab, welche die Seele hat. Wenn ich Schmerzen für ein Übel halte, dann wird sich mir die Tatsache, dass mich mein Finger schmerzt, anders darstellen als wenn ich Schmerz, wie ein Weiser, für etwas völlig Indifferentes halte. Der Weise und der Nichtweise haben ganz verschiedene Vorstellungen von denselben Dingen. Während Schmerz bei uns

29 Siehe auch Enn. IV 3, 32; VI 8, 18, 19. 
(mittels einer Vorstellung) die Seele in Aufruhr versetzen und so, wenn wir zustimmen, Handlungen auslösen kann, wird dies beim Weisen nicht der Fall sein. ${ }^{30}$ Denn der Weise weiß, dass Schmerz ein Affekt des Leibes ist. Er wird ihn zur Kenntnis nehmen, ohne aber selbst (d. h. in seiner Seele) emotional davon betroffen zu sein. Er wird also den Schmerz als Schmerz des Leibes und somit als äußerlich wahrnehmen.

Bevor wir abschließend kurz zur Klasse von jenen Emotionen kommen, die keine leibliche Basis haben, seien zwei zentrale Aspekte von Plotins Emotionstheorie, soweit sie uns Nichtweise betreffen, betont. Da Emotionen bei Plotin wie bei der Stoa propositionalen Gehalt haben, findet sich auch bei Plotin ein Element, das vielen an der stoischen Position attraktiv erscheint. In dieser Hinsicht ist Plotins Emotionstheorie also kognitivistisch. Anders als die Stoa glaubt Plotin aber, dass die Emotionen der gewöhnlichen, nichtweisen Menschen ihre qualitative Basis in einem leiblichen Affekt haben, ohne jedoch auf solche Affekte reduziert werden zu können. Plotin kann so im Gegensatz zur Stoa den engen Zusammenhang zwischen leiblichen und emotionalen Zuständen einer Person erklären, ohne eine nichtkognitivistische Position einzunehmen. Dieser Punkt, mit dem er über die Stoa hinausgeht, scheint mir ein zweites attraktives Element an Plotins Emotionstheorie zu sein.

Der Fokus unserer Diskussion lag auf den auf dem Leib basierenden Emotionen des gewöhnlichen Menschen. Wie steht es mit dem Weisen? Hat er, der von den Affekten des Leibes ganz unbeeinflusst bleibt, keine Emotionen? Wir wissen, dass die Stoa ihren Weisen nicht ohne Emotionen leben lässt. ${ }^{31}$ Die Emotionen des Weisen sind allerdings grundverschieden von denen der gewöhnlichen Leute - sie sind sogenannte gute Emotionen (eupatheiai). Plotins Auffassung ist ähnlich. Sein Weiser ist frei von Emotionen, die qualitativ auf leiblichen Affekten beruhen. Trotzdem schreibt auch Plotin dem Weisen Emotionen zu, Emotionen anderer Art allerdings. Die Emotionen des Weisen verdanken ihre motivationale Kraft und ihre Gefühlsfärbung nicht dem Leib, sondern der Vernunft. So beschert dem Weisen das Nachdenken über die Wirklichkeit höchste Befriedigung (Enn. IV 7, 30). Aber der Weise ist nicht nur in einem guten emotionalen Zustand, wenn er über

30 Dazu, dass sich die Vorstellungen des Weisen fundamental von denen des Nichtweisen unterscheiden, siehe Enn. I 4, 7-8 mit Schniewind 2003, 148-150.

31 Frede 1986. 
die Wirklichkeit nachdenkt, sondern selbst dann, wenn er sich um die profanen irdischen Angelegenheiten kümmert: „Heiter aber ist der Weise immerdar, sein Zustand ist ruhevoll, seine Stimmung voll Zufriedenheit, und keines der angeblichen Übel kann sie erschüttern, wenn er wirklich ein Weiser ist.“"32

\section{Literatur}

Plotins Schriften werden nach der von Henry und Schwyzer besorgten Ausgabe Plotini Opera zitiert - vollständige Angaben siehe unten. Alle Zitate werden in der Übersetzung von Harder (mit leichten Modifikationen des Verfassers) wiedergegeben. Üblicherweise ist die Ausgabe der Schriften Plotins in sechs Neunergruppen, sogenannten Enneaden, eingeteilt. Die Zahl in römischen Ziffern verweist auf die Enneade, die folgende Zahl in arabischen Ziffern auf die Stellung des Traktats innerhalb der Neunergruppe. Die verwendete Sigle ist: Enn.

Arthur, E. P. (1983), The Stoic Analysis of the Mind's Reactions to Presentations, in: Hermes 111, 69-78.

Blumenthal, Henry J. (1971), Plotinus' Psychology. His Doctrines of the Embodied Soul, Den Haag.

Caluori, Damian (2005), The Essential Functions of a Plotinian Soul, in: Rhizai 2, 7593.

Cooper, John (1984), Plato's Theory of Human Motivation, in: History of Philosophy Quarterly 1, 3-24.

Des Chene, Dennis (2001), Spirits and Clocks. Machine and Organism in Descartes, Ithaca.

Dillon, John (1983), Metriopatheia and Apatheia: Some Reflections on a Controversy in Later Greek Ethics, in: John P. Anton/Anthony Preus (Hg.), Essays in Ancient Greek Philosophy II, Albany, 508-517.

Emilsson, Eyjólfur Kjalar (1988), Plotinus on Sense-Perception: A Philosophical Study, Cambridge.

Emilsson, Eyjólfur Kjalar (1998), Plotinus on the Emotions, in: Juha Sihvola/Troels Engberg-Pedersen (Hg.), The Emotions in Hellenistic Philosophy, Dordrecht, 339-363.

Frede, Michael (1983), Stoics and Sceptics on Clear and Distinct Impressions, in: Myles Burnyeat (Hg.), The Skeptical Tradition, Berkeley, 65-93.

Frede, Michael (1986), The Stoic Doctrine of the Affections of the Soul, in: Malcolm Schofield/Gisela Striker (Hg.), The Norms of Nature. Studies in Hellenistic Ethics, Cambridge, 93-110.

Giglioni, Guido (1995), Automata compared: Boyle, Leibniz and the Debate on the Notion of Life and Mind, in: British Journal for the History of Philosophy 3, 249-278.

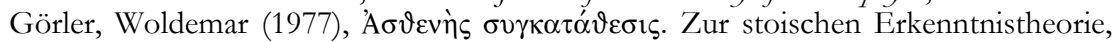
in: Würzburger Jahrbücher für Altertumswissenschaft, N. F. III, 83-92.

Igal, Jesús (1979), Aristóteles y la evolución de la antropología de Plotino, in: Pensiamento 35, 315-345.

Kristeller, Paul Oskar (1929), Der Begriff der Seele in der Ethik des Plotin, Tübingen.

32 Ich danke Sophie Caflisch und Magdalena Hoffmann herzlich für ihre hilfreichen Bemerkungen zu einer früheren Fassung dieses Aufsatzes. 
Nussbaum, Martha C. (2001), Upheavals of Thought. The Intelligence of Emotions, Cambridge.

Plotin (1956-1971), Plotins Schriften. Neubearbeitung mit griechischem Lesetext und Anmerkungen, hrsg. von Richard Harder/Rudolf Beutler/Willy Theiler, Hamburg.

Plotin (1964-1982), Plotini Opera, hrsg. von Paul Henry und Hans-Rudolf Schwyzer, Oxford (=Enn.).

Plotinus (1995), Plotinus Ennead III.6. On the Impassivity of the Bodiless. Translation and Commentary by Barry Fleet, Oxford.

Rehm, David (1997), The Structure of Emotions in Plotinus, in: American Catholic Philosophical Quarterly 71, 469-488.

Schniewind, Alexandrine (2003), L’éthique du sage chez. Plotin. Le paradigme du spoudaios, Paris.

Schwyzer, Hans-Rudolf (1960), „Bewusst“ und „Unbewusst“ bei Plotin, in: Les sources de Plotin. Entretiens sur l'antiquité classique 5 , Genève.

Smith, Andrew (1999), The Significance of Practical Ethics for Plotinus, in: John J. Cleary (Hg.), Traditions of Platonism: Essays in Honour of John Dillon, Aldershot, 227236. 